\title{
RIQUIER PROBLEM IN A BIHARMONIC SPACE
}

\author{
MOUSTAFA K. DAMLAKHI
}

\begin{abstract}
After defining the notion of a biharmonic space $\Omega$ which generalizes $\mathbb{R}^{n}$, Riemann surfaces and Rimannian manifolds, we discuss the Riquier problem for an open set in $\Omega$ provided with the Wiener boundary.
\end{abstract}

\section{Introduction}

The Riquier problem in $\mathbb{R}^{n}$ is to find a biharmonic function $b$ on an open set $\omega$ such that $b$ and $\Delta b$ tend respectively to previously given continuous functions $f$ and $g$ on the Euclidean boundary $\partial \omega$ in $\mathbb{R}^{n}$. The connection between the Riquier problem and the Dirichlet problem is easy to understand.

In this note, we discuss this problem in the general context of a biharmonic space. First we define the notion of a biharmonic space $\left(\omega, H, H^{*}, \lambda\right)$ where $\omega$ is a locally compact space provided with two harmonic sheaves $H$ and $H^{*}$ satisfying the three axioms of Brelot [2] and $\lambda$ is a fixed Radon measure on $\omega$. According to this definition, $\mathbb{R}^{n}$ for all $n \geq 1$, Riemann surfaces, Riemannian manifolds and domains $\omega$ in $\mathbb{R}^{n}$ with the solutions of certain elliptic differential operators of order 2 as harmonic functions on $\omega$ are all examples of biharmonic spaces.

The final section considers the solution to the Riquier problem on a relatively compact domain $\omega$ in a biharmonic space, where $\omega$ is endowed with the Wiener boundary.

\section{Riquier Problem in Riemann Spaces}

A locally integrable function $b$ defined on an open set $\omega$ in $\mathbb{R}^{n}, n \geq 2$, is said to be biharmonic if and only if $\Delta^{2} b=0$ in the sense of distributions. It is known that if $\Delta^{2} b=0$, there exists a $C^{\infty}$-function $b_{1}$ on $\omega$ such that $b=b_{1}$ a.e.. Consequently we always assume that a biharmonic function is a $C^{\infty}$-function.

We shall say that $Q_{y}(x)>0$ is the biharmonic Green potential on $\omega$, with pole $y$, if $\Delta^{2} Q_{y}=\delta_{y}$ where $\delta_{y}$ is the Dirac measure. Note that $\Delta Q_{y}(x)=-G_{y}(x)$ where $G_{y}(x)=G^{\omega}(x, y)$ is the symmetric Green function on $\omega$ with pole $y$. It is shown in [1] that the biharmonic Green potentials exist on an arbitrary domain in $\mathbb{R}^{n}$, if $n \geq 5$;

Received November 14, 2001; revised July 1, 2002.

2000 Mathematics Subject Classification. 31D05, 31B25.

Key words and phrases. Riquier problem, Wiener boundary. 
however if $\omega$ is a relatively compact domain in $\mathbb{R}^{n}, n \geq 2$, then the biharmonic Green potentials exist on $\omega$.

A relatively compact domain $\omega$ in $\mathbb{R}^{n}$ is said to be regular for the Dirichlet problem, if given a finite continuous function $f$ on $\partial \omega$ there exists a harmonic function $H_{f}^{\omega}(x)$ on $\omega$ such that $\lim _{x \rightarrow y} H_{f}^{\omega}(x)=f(y)$ for every $y \in \partial \omega$. The Riquier problem for a relatively compact open set $\omega$ in $\mathbb{R}^{n}$ is the following: Given two finite continuous functions $f$ and $g$ on $\partial \omega$, find a biharmonic function $b$ on $\omega$ such that $\lim _{x \rightarrow y} b(x)=f(y)$ and $\lim _{x \rightarrow y} \Delta b(x)=g(y)$ for every $y$ in $\partial \omega$.

Solution to the Riquier problem. Let $\omega$ be a relatively compact domain in $\mathbb{R}^{n}$. If $\omega$ is regular for the Dirichlet problem and if $f$ and $g$ are two finite continuous functions on $\partial \omega$, then there exists a biharmonic function $b$ on $\omega$ such that $b$ tends to $f$ and $\Delta b$ tends to $g$ on $\partial \omega$.

Proof. Let $X$ be a relatively compact domain in $\mathbb{R}^{n}$ such that $\bar{\omega} \subset X$. Since $\omega$ is regular for the Dirichlet problem, there exists a unique continuous function $H_{g}^{\omega}$ on $\bar{\omega}$ such that $H_{g}^{\omega}=g$ on $\partial \omega$ and $H_{g}^{\omega}$ is harmonic on $\omega$.

Let $g_{1}$ be a finite continuous function on $X$ with compact support such that $g_{1}=$ $-H_{g}^{\omega}$ on $\bar{\omega}$. Since $X$ is relatively compact, the harmonic Green potential $G^{X}(x, y)$ is defined on $X$. Let $u(x)=\int_{X} G^{X}(x, y) g_{1}(y) d y$. Then $u(x)$ is finite continuous on $X$ and on $\omega, \Delta u=\Delta\left(G^{X} \star g_{1}\right)=-\delta \star g_{1}=-g_{1}$, and hence $\Delta^{2} u=0$ on $\omega$. Define on $\omega, b=u-H_{u}^{\omega}+H_{f}^{\omega}$. Then $b$ is biharmonic on $\omega$ such that $b$ tends to $f$ on $\partial \omega$ and $\Delta u$ tends to $-g_{1}=H_{g}^{\omega}=g$ on $\partial \omega$.

The above problem has a counterpart in a Riemannian manifold. Let $R$ be an oriented Riemannian manifold of dimension $\geq 2$ with local parameters $x=\left(x^{1}, \ldots, x^{n}\right)$ and a $C^{\infty_{-}}$ metric tensor $g_{i j}$ such that $g_{i j} x^{i} x^{j}$ is positive definite. If $g$ is the determinant of $g_{i j}$, let us denote the volume element by $d x=g^{\frac{1}{2}} d x^{1} \cdots d x^{n} ; \Delta=d \delta+\delta d$ denotes the LaplaceBeltrami operator which is also defined as $\Delta f=-d i v$ grad $f$. In local coordinates, $\Delta$ has an invariant expression $\Delta f=-g^{\frac{1}{2}} \frac{\partial}{\partial x^{i}}\left(g^{\frac{1}{2}} g^{i j} \frac{\partial f}{\partial x^{j}}\right)$ and in the Euclidean case this reduces to $\Delta f=-\sum_{i=1}^{n} \frac{\partial^{2} f}{\partial x^{i 2}}$.

A function $f$ is called harmonic (resp. biharmonic) if $\Delta f=0$ (resp. $\left.\Delta^{2} f=0\right)$. Then we can solve the Riquier problem in $R$ as we did in the Euclidean case $\mathbb{R}^{n}$. The result is as follows: Let $\omega$ be a relatively compact domain in a Riemannian manifold $R$ as above. Suppose $\omega$ is regular for the Dirichlet problem. Then given a pair of finite continuous functions $f$ and $g$ on $\partial \omega$, there exists a unique biharmonic function $b$ on $\omega$ such that $\lim _{x \rightarrow y} b(x)=f(y)$ and $\lim _{x \rightarrow y} \Delta b(x)=g(y)$ for every $y \in \partial \omega$.

However this procedure cannot be carried out in a Riemann surface. The difficulty arises from the fact that the Laplacian is not conformally invariant under a parametric change and hence the definition of a biharmonic function on a Riemann surface as a function $b$ such that $\Delta^{2} b=0$ is not acceptable. To overcome this problem, we shall introduce the biharmonic functions on a Riemann surface using the following lemma (see Anandam [1]). 
Lemma 2.1. Let $\mu$ be a Radon measure on a domain $\omega$ in a Riemann surface $R$. Then there exists a superharmonic function $s$ on $\omega$ such that the measure associated to $s$ in a local Riesz representation is $\mu$.

Proof. Write $\omega=\cup_{n=1}^{\infty} K_{n}$ where $K_{n}$ is compact, $K_{n} \subset \stackrel{0}{K}_{n+1}$ and each component of $\omega \backslash K_{n}$ is not relatively compact. For $n \geq 1$, let $\mu_{n}$ be the restriction of $\mu$ to $K_{n+1} \backslash K_{n}$ and let $\mu_{0}$ be the restriction of $\mu$ to $K_{1}$.

Since $\mu_{n}$ has compact support, there exists a superharmonic function $s_{n}$ on $\omega$ with associated measure $\mu_{n}$ in a local Riesz representation. By the approximation theorem of Pfluger [7] p.192, there exists a harmonic function $v_{n}$ on $\omega, n \geq 1$, such that $\mid s_{n+1}-$ $v_{n+1} \mid<\frac{1}{2^{n}}$ on $K_{n}$. Set $v_{0}=v_{1}=0$. Define $s=\sum_{n=0}^{\infty}\left(s_{n}-v_{n}\right)$ on $\omega$. Then $s$ is a superharmonic function on $\omega$. For, let $K$ be any compact set in $\omega$; then $K \subset \stackrel{0}{K}$ for $m \geq 2$. Write $s=\sum_{n=0}^{m}\left(s_{n}-v_{n}\right)+\sum_{n=m+1}^{\infty}\left(s_{n}-v_{n}\right)=s_{1}+s_{2}$.

Here $s_{2}$ is harmonic on $\stackrel{0}{K}_{m}$ and the measure associated with $s_{1}$ on $K$ is $\mu$. Thus $s$ is superharmonic on a neighbourhood of $K$ with associated measure $\mu$ on $K$. Since $K$ is an arbitrary compact set in $\omega, s$ is superharmonic on $\omega$ with $\mu$ as its assoicated measure in a local Riesz representation.

Notation. If $\omega$ is an open set in a Riemann surface $R$ and if $\mu$ is a Radon measure on $\omega$, let $s$ be a superharmonic function on $\omega$ with associated measure $\mu$ constructed as in Lemma 2.1. Note that $s$ is constructed only up to an additive harmonic function on $\omega$. Let us write $L s=-\mu$ on $\omega$ to denote that $s$ is a superharmonic function on $\omega$ with $\mu$ as its associated measure in a local Riesz represention. Let $d x$ be the surface measure on $R$ and $f$ be a locally $d x$-integrable function on an open set $\omega$. We write $L u=-f$ on $\omega$ to denote that $u$ is a $\delta$-superharmonic function on $\omega$ with associated signed measure $\lambda$ defined by $d \lambda=f^{+} d x-f^{-} d x$.

With these notations, we shall say a function $b$ defined on an open set $\omega$ in $R$ is biharmonic if there exists a harmonic function $h$ on $\omega$ such that $L b=-h$ on $\omega$. Clearly this way of defining a biharmonic function $b$, if carried out on a Riemannian monifold, coincides with the previous definition of $\Delta^{2} b=0$. Thus, the notion of biharmonic functions can be extended to a Riemann surface without the intervention of the Laplace operator $\Delta$, and the solution to the Riquier problem can be sought in this case also.

This method suggests that biharmonic functions can also be considered in a general locally compact space $\omega$ provided with a sheaf of harmonic functions, where the notion of the derivatives does not exist.

\section{Riquier Problem in a Harmonic Space}

We shall consider now the Riquier problem in a very general set up that englobes the different cases mentioned in the previous section.

Let $\Omega$ be a connected locally compact space, with a countable base, but $\Omega$ is not compact. Let $H$ and $H^{*}$ be two sheaves on $\Omega$ satisfying the axioms 1, 2, 3 of M. Brelot 
[2]. Assume the constants are harmonic in $H$ and $H^{*}$. There may or may not exist any potential $>0$ in $H$ or $H^{*}$. (That is, in the harmonic classification of Rimannian spaces, $H$ and $H^{*}$ can be hyperbolic or parabolic). But we know that in any domain $\omega$ in $\Omega$, there exists an $H$-potential if and only if $\Omega \backslash \omega$ is not locally polar in the $H$-sheaf (with a similar result for the $H^{*}$-sheaf). We assume three more things on the $H$-sheaf only:

1. $H$ satisfies the local axiom of proportionality. That is, if $\omega$ is a relatively compact domain, $y \in \omega$, and if $p$ and $q$ are two $H$-potentials on $\omega$ with harmonic point support $y$, then $p$ and $q$ are proportional.

2. With the above assumption, in any domain $\omega$ such that $\Omega \backslash \omega$ is not locally polar, there always exists a Green $H$-potential $G(x, y)$. We assume that such a function is symmetric.

3. If $\omega$ is a domain and if $h$ is an $H$-harmonic function on $\omega$ such that $h=0$ on a neighbourhood of a point in $\omega$, then $h \equiv 0$ on $\omega$.

Definition 3.1. Let the above assumptions on $\Omega, H$ and $H^{*}$ be satisfied. Let us fix a Radon measure $\lambda \geq 0$ on $\omega$. Then we call $\left(\Omega, H, H^{*}, \lambda\right)$ a biharmonic space.

It appears that we have to assume too many things to define a biharmonic space $\left(\Omega, H, H^{*}, \lambda\right)$. But the fact that these conditions are satisfied in the following cases is reassuring.

1. $\Omega=\mathbb{R}^{n} ; n \geq 1$, with the harmonic sheaves $H=H^{*}$ defined as usual by means of the Laplacian; $\lambda$ is the Lebesgue measure.

2. Riemann surfaces and Riemannian manifolds with the local definition of harmonic functions, $\lambda$ is the surface or the volume measure.

3. Let $\Omega$ be a domain in $\mathbb{R}^{n}$. Let

$$
L=\sum_{i, j} a_{i j} \frac{\partial^{2}}{\partial x_{i} \partial x_{j}}+\sum_{i} b_{i} \frac{\partial}{\partial x_{i}}
$$

be an elliptic differential operator of order $2 ; a_{i j}=a_{j i}$ are of $C^{2, \lambda}$ class and $b_{i}$ are of $C^{1, \lambda}$ class (here $C^{k, \lambda}$ denotes the class of functions $f$ which are $k$-times continuously differentiable with their $k$-th order partial derivatives benig locally Lipschitzien); and the quadratic form $\sum_{i, j} a_{i j} \xi_{i} \xi_{j}$ is positive definite for all $x$ in $\Omega$. Then, the class of $C^{2}$ functions $u$ in $\Omega$ satisfying the equation $L u=0$ form a harmonic sheaf $H$ in the sense of Brelot (see Hervé [5]). Let $H^{*}$ be the harmonic sheaf on $\Omega$ defined by a similar elliptic differential operator $L^{*}$. Then $\left(\Omega, H, H^{*}, \lambda\right)$ is a biharmonic space, with $\lambda$ the Lebesgue measure.

Theorem 3.2. Let $(\Omega, H)$ be a harmonic space with the sheaf $H$ satisfying the above-mentioned bypotheses. Then, given a Radon measure $\mu$ on an open set $\omega$ in $\Omega$, there exists an $H$-superharmonic function $s$ on $\omega$, denoted by $L s=-\mu$, such that the measure associated with $s$ in a local Riesz representation is $\mu$.

Proof. To prove this theorem, we follow the method given in the proof of Lemma 2.1 , just replacing the approximation theorem of Pfluger by Theorem 10 of De la Pradelle $[4]$. 
Notation. Let $\omega$ be an open set in a biharmonic space $\left(\Omega, H, H^{*}, \lambda\right)$. Then given any continuous function $f$ on $\omega$, by Theorem 3.2, there exists a $\delta$-superharmonic function $u$ on $\omega$ with respect to the harmonic sheaf $H$ such that the measure associated with $u$ in a local Riesz representation is $\mu$, given by $d \mu=f d \lambda$. We always assume that one such function $u$ is continuous on $\omega$ and denote its dependence on $f$ by the notation $L u=-f$.

Definition 3.3. Let $\omega$ be an open set in a biharmonic space $\left(\Omega, H, H^{*}, \lambda\right)$. Then given an $H^{*}$-harmonic function on $\omega$, there exists a $\delta$-superharmonic function $b$ on $\omega$ with respect to the harmonic sheaf $H$ with associated signed measure $\mu$ given by $d \mu=h^{*} d \lambda$; that is $L b=-h^{*}$ on $\omega$. We say that $b$ is biharmonic on $\omega$.

Theorem 3.4. Let $\left(\Omega, H, H^{*}, \lambda\right)$ be a biharmonic space. Let $\omega$ be a relatively compact domain with its boundary $\partial \omega$ regular for the Dirichlet problem in the two harmonic spaces $(\Omega, H)$ and $\left(\Omega, H^{*}\right)$. Then given two finite continuous functions $f$ and $g$ on $\partial \omega$, there exists a unique $H$-biharmonic function $b$ on $\omega$ such that $\lim _{x \rightarrow y} b(x)=f(y)$ and $\lim _{x \rightarrow y} L b(x)=g(y)$ for every $y \in \partial \omega$.

Proof. Let $h^{*}$ be the unique $H^{*}$-harmonic function on $\omega$ tending to $-g$ on $\partial \omega$. Let $\omega_{0}$ be a relatively compact domain such that $\omega_{0} \supset \bar{\omega}$. Extend the function $h^{*}$ as a continuous function $g_{1}$ on $\omega_{0}$. Let $L u=-g_{1}$ on $\omega_{0}$. By hypothesis, $u$ is continuous on $\omega_{0}$. Let $u_{1}$ and $f_{1}$ be $H$-harmonic functions on $\omega$ tending to $u$ and $f$ respectively. Let $b(x)=u(x)-u_{1}(x)+f_{1}(x)$. Then $b$ is biharmonic on $\omega$, tending to $f$ on $\partial \omega ;$ moreover on $\omega, L b=L u=-g_{1}=-h^{*}$ so that $L b$ tends to $g$ on $\partial \omega$.

\section{Riquier Problem with Wiener Boundary}

Let $R$ be a Riemannian manifold and $\omega$ a relatively compact domain in $R$. Let $\bar{\omega}$ be the Wiener compactification of $\omega$ and let $\partial \omega=\bar{\omega} \backslash \omega$. Suppose $f$ and $g$ are two finite continuous function on $\partial \omega$. Since $\partial \omega$ is resolutive, $h=H_{-f}^{\partial \omega}$ is a bounded harmonic function on $\omega$. If $G(x, y)$ is the Green function on $\omega, G \in L^{1}(\omega)$ and hence if $u(x)=\int_{\omega} G(x, y) h(y) d y, u(x)$ is bounded and $\Delta u=-h$; since $h$ is in $C^{\infty}(\omega)$, we can assume $u$ also is in $C^{\infty}(\omega)$.

Recall (Proposition 4.7[6]) that if $\mu$ is a measure in $\omega$ with $\|\mu\|$ finite, then $\int G(x, y)$ $d \mu(y)$ is a potential on $\omega$. Hence $u(x)=\int_{\omega} G(x, y) h^{+}(y) d y-\int_{\omega} G(x, y) h^{-}(y) d y$ is the difference of two potentials and hence harmonizable.

Thus $u$ is a bounded Wiener function on $\omega$ and consequently $u$ extends as a continuous function on the Wiener compactification $\bar{\omega}$.

Let $v=H_{g-u}^{\partial \omega}$. Then $v$ is bounded harmonic on $\omega$ and $b=u+v$ is a bounded biharmonic function on $\omega, \Delta b=-h, b$ tending to $g$ and $\Delta b$ tending to $f$ at the regular points of $\partial \omega$ (see Section 4 Chapter VIII [8]).

Proceeding in the same way, we prove the following theorem in the axiomatic case where the theory of Wiener compatification is due to Constantinescu and Cornea [3].

Theorem 4.1. Let $\left(\Omega, H, H^{*}, \lambda\right)$ be a biharmonic space. Let $\omega$ be a relatively compact domain in $\Omega$. Let $\Gamma$ and $\Gamma^{*}$ be the Wiener harmonic boundaries of $\omega$ in $(\omega, H)$ and $\left(\omega, H^{*}\right)$ 
respectively. Then if $g$ and $f^{*}$ are finite continuous on $\Gamma$ and $\Gamma^{*}$ respectively, there exists a unique biharmonic function $b$ on $\omega$ such that $b$ and $L b$ are bounded, $b$ tends to $g$ on $\Gamma$ and $L b$ tends to $f^{*}$ on $\Gamma^{*}$.

Proof. Extend $f^{*}$ and $g$ as finite continuous functions on the Wiener boundaries $\partial \omega^{*}$ and $\partial \omega$ respectively. Let $h^{*}=H_{-f^{*}}^{\partial \omega^{*}}$ on $\omega^{*}$. Let $\omega_{1}$ be an $H$-regular domain containing $\omega_{c}$, the closure of $\omega$ in $\Omega$. By the assumption on the continuity of the biharmonic functions, there exists a finite continuous function $u$ on $\omega_{1}$ such that $L u=-1$; that is if $G_{\omega_{1}}(x, y)$ is the symmetric $H$-Green kernel on $\omega_{1}, u(x)=\int_{\omega_{1}} G_{\omega_{1}}(x, y) d \lambda(y)$ is finite continuous on $\omega_{1}$. Since $u$ is finite continuous on $\omega_{1} \supset \omega_{c}, u$ is bounded on $\omega_{c}$.

Hence, for $x \in \omega$,

$$
\int_{\omega} G_{\omega}(x, y) d \lambda(y) \leq \int_{\omega} G_{\omega_{1}}(x, y) d \lambda(y) \leq \int_{\omega_{1}} G_{\omega_{1}}(x, y) d \lambda(y)=u(x) .
$$

Consequently, since $h^{*}$ is bounded on $\omega$ and since $G_{\omega}(x, y)$ is symmetric by hypothesis, $\int_{\omega} G_{\omega}(x, y) h^{+*}(y) d \lambda(y)$ and $\int_{\omega} G_{\omega}(x, y) h^{-*}(y) d \lambda(y)$ are well defined bounded $H^{*}$-potentials on $\omega$; hence $v(x)=\int_{\omega} G_{\omega}(x, y) h^{*}(y) d \lambda(y)$ is the difference of two bounded potentials and hence harmonizable; also $v$ benig biharmonic, is continuous. Thus $v$ is a bounded Wiener function on $\omega$ and hence $v$ extends continuously on the Wiener compactification $\bar{\omega}$.

Since $g$ is a finite continuous function on $\partial \omega$, there exists a bounded $H$-harmonic $h_{1}$ on $\omega$ tending to $g-v$ on $\Gamma$. Let $b=v+h_{1}$ on $\omega$. Then $b$ is a bounded biharmonic function on $\omega$ such that $b$ tends to $g$ on $\Gamma$ and $L b=-h^{*}$ tends to $f^{*}$ on $\Gamma^{*}$.

For the uniqueness of $b$, notice that if $u$ is bounded on $\omega$ and if $L u$ is bounded on $\omega^{*}$ such that $u$ and $L u$ tend to 0 on $\Gamma$ and $\Gamma^{*}$ respectively, then $u \equiv 0$. For, $L u$ is bounded $H^{*}$-harmonic on $\omega$ and tends to 0 on $\Gamma^{*}$ and hence $L u \equiv 0$; this means that $u$ is bounded $H$-harmonic on $\omega$ and tends to 0 on $\Gamma$ and hence $u \equiv 0$.

\section{Acknowledgement}

The author thanks the College of Science Research Center, King Saud University, for the grant MATH/1420/20.

\section{References}

[1] V. Anandam, Biharmonic classification of harmonic spaces, Revue Roumaine Math. Pures et Appliquées 45(2000), 383-395.

[2] M. Brelot, Axiomatique des fonctions harmoniques, Les Presses de 1'Université de Montréal, 1966.

[3] C. Constantinescu and A. Cornea, Compactification of harmonic spaces, Nagoya Math. J. 25(1965), 1-57.

[4] A. De la Pradelle, Approximation et caractère de quasi-analyticité dans la théorie axiomatique des fonctions harmoniques, Ann. Inst. Fourier 17(1967), 383-399. 
[5] R. M. Hervé, Recherches axiomatiques sur la théorie des fonctions surharmoniques et du potentiel, Ann. Inst. Fourier 12(1962), 415-571.

[6] F. Y. Maeda, Dirichlet integrals on harmonic spaces, L. N. Maths. 803, Springer-Verlag, 1980.

[7] A. Pfluger, Theorie der Riemannsher Flächen, Springer-Verlag, 1957.

[8] L. Sario, M. Nakai, C. Wang and L. Chung, Classification theory of Riemannian manifolds, L. N. Maths. 605, Springer-Verlag, 1977.

Department of Mathematics, College of Science, King Saud University, P.O.Box 2455, Riyadh 11451, Saudi Arabia.

E-mail: damlakhi@ksu.edu.sa 